\title{
LESÃO COMPLEXA DA VIA BILIAR PRINCIPAL: A LIGADURA COMO OPÇÃO ASSOCIADA A COLECISTOJEJUNOSTOMIA
}

\section{COMMON BILE DUCT INJURY: LIGATION AND CHOLECYSTOJEJUNOSTOMY AS SURGICAL OPTION}

\author{
Sizenando Vieira Starling, TCBC-MG ${ }^{1}$ \\ Wilson Luís Abrantes, TCBC-MG ${ }^{2}$
}

\section{INTRODUÇÃO}

As lesões da via biliar principal (VBP) por trauma externo são $\operatorname{raras}^{1}$. Geralmente são provocadas por trauma penetrante ${ }^{2,3}$ e vem quase sempre acompanhadas de outras lesões intraabdominais ${ }^{1}$. $\mathrm{O}$ tratamento pode ser feito por várias técnicas. $\mathrm{O}$ objetivo em todas elas é conduzir a bile ao intestino, seja pela reconstituição anatômica do canal ou mediante anastomose bíliodigestiva (hepático ou coledocojejunostomia). A dimensão reduzida do canal ${ }^{4}$ sempre dificulta a confecção segura da anastomose, favorecendo o aparecimento de complicações pós-operatórias precoces (fístula biliar) ou tardias (estenoses $)^{1,2}$. Nas lesões complexas e distais à desembocadura do duto cístico uma alternativa segura é a ligadura da VBP associada a uma colecistojejunostomia.

\section{RELATO DO CASO}

Paciente de 22 anos, vítima de agressão por arma branca no hipocôndrio esquerdo, com sinais evidentes de irritação peritonial. À laparotomia foram encontradas: lesão transfixante de antro gástrico, lesão transfixante de cabeça de pâncreas com lesão do ducto pancreático principal, secção total do colédoco intrapancreático e lesão única da segunda porção duodenal. Tratamento realizado: gastrorrafia e duodenorrafia em dois planos, secção da cabeça do pâncreas na altura da lesão com sutura do coto proximal e confecção de pancreatojejunostomia términoterminal em Y de Roux (Figura 1). Em seguida procedeu-se à ligadura da VBP abaixo da desembocadura do cístico e realização de colecistojejunostomia látero-lateral na alça do Y de Roux (Figura 2). O paciente evoluiu com quadro de fístula pancreática de baixo débito com fechamento espontâneo no oitavo dia de pós-operatório. Recebeu alta no décimo sétimo dia de pós-operatório e está sob controle ambulatorial.

\section{DISCUSSÃO}

As lesões da VBP por trauma externo, embora raras, são de tratamento desafiador. Ocorrem em menos de $5 \%$ de todos os traumatismos abdominais ${ }^{3}$. Podem ser causadas por traumatismos abertos ou fechados. Nos traumas fechados a localização mais freqüente é na junção com o pâncreas ${ }^{4}$.

As lesões da VBP são classificadas como simples (lacerações parciais com menos de $50 \%$ da circunferência) ou complexas (lacerações parciais com mais de $50 \%$ da circunferência, secção total e perda de segmento da parede ductal). Estas últimas são de reparo difícil e com alto índice de complicação.

1. Cirurgião Titular do Hospital João XXIII (FHEMIG), do Serviço Médico de Urgência do IPSEMG e do Hospital Evangélico de Belo Horizonte. MG.

2. Consultor de Cirurgia do Hospital João XXIII (FHEMIG) e Chefe do Serviço de Cirurgia Geral do Hospital Felício Rocho. Belo Horizonte. MG 


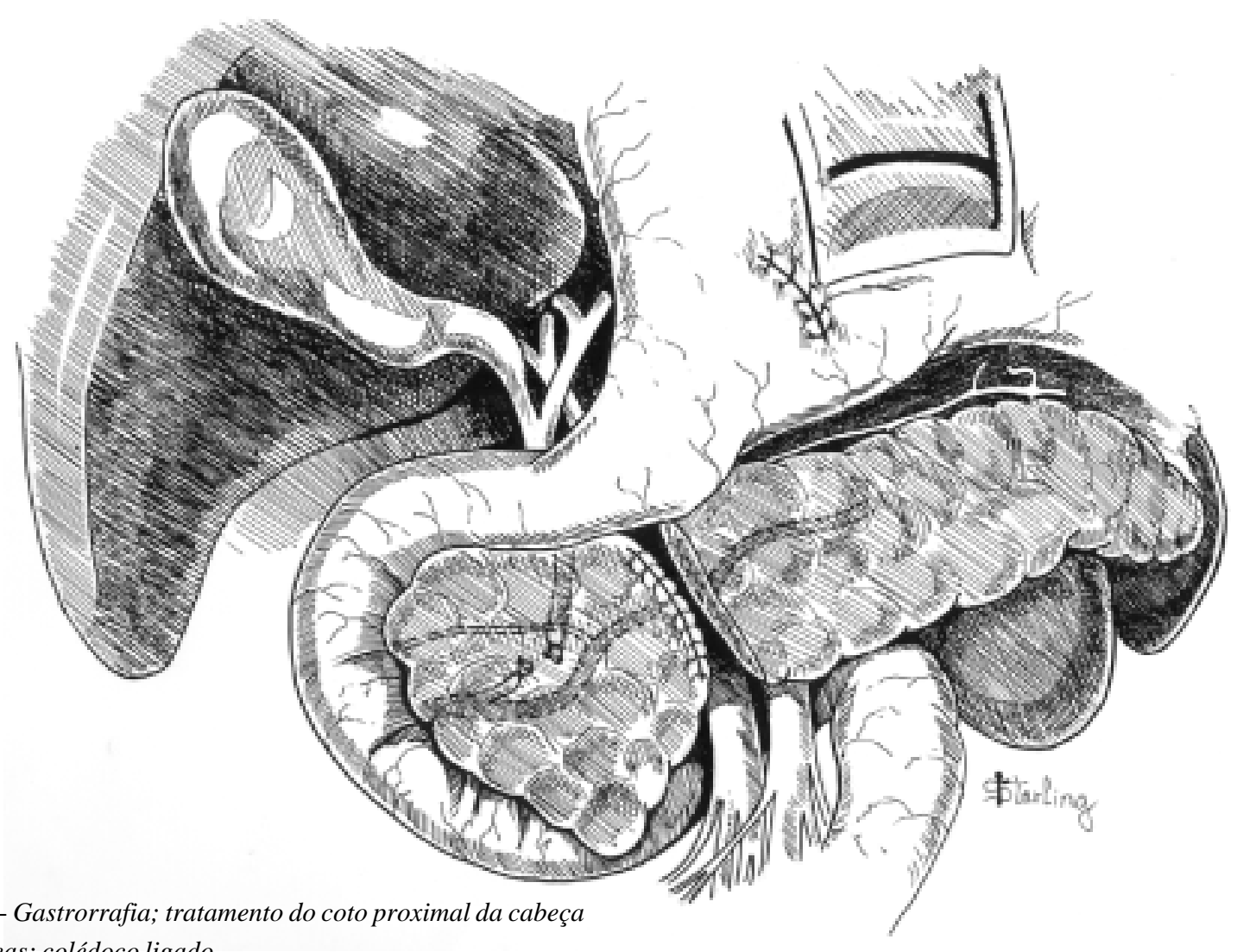

Figura 1 - Gastrorrafia; tratamento do coto proximal da cabeça do pâncreas; colédoco ligado.

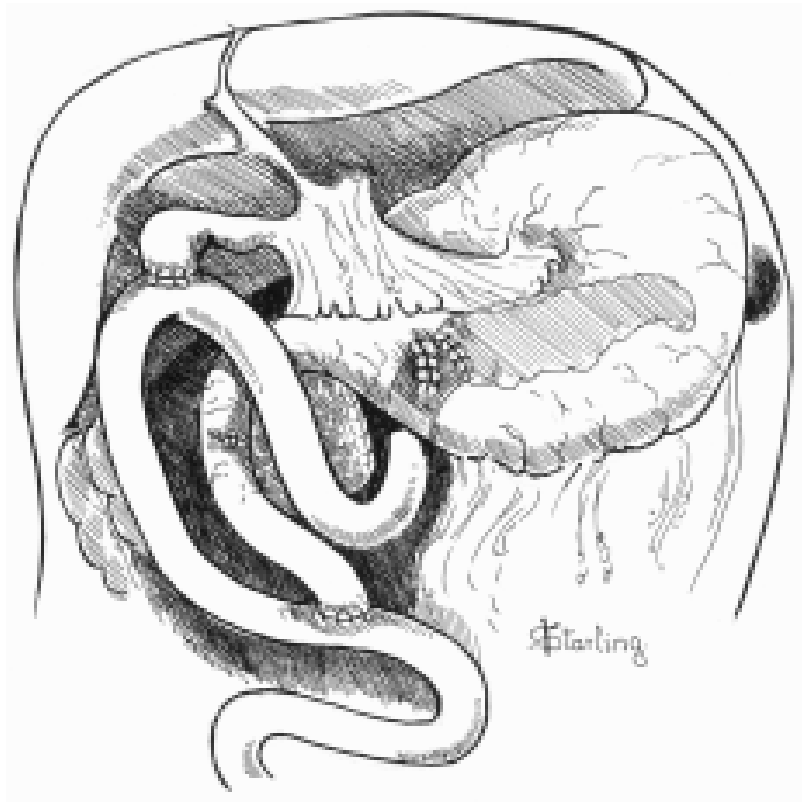

Figura 2 - Aspecto final: duodenorrafia, pancreatojejunostomia e colecistojejunostomia em Y de Roux.
Nos traumas penetrantes o diagnóstico é feito no per-operatório pelo achado de bile livre no abdome ou impregnada aos tecidos adjacentes. Nos traumas fechados com única lesão da VBP, o diagnóstico imediato é difícil porque a bile, na ausência de infecção, produz pouca irritação peritonial $^{3,4}$. Geralmente, na maioria dos casos, a lesão da VBP encontra-se associada à outras lesões abdominais principalmente hepática, vasculares, duodenais e pancreáticas ${ }^{3,4}$.

A escolha do tratamento depende dos seguintes fatores: local e tipo da lesão, presença de lesões associadas, instabilidade hemodinâmica e calibre da VBP ${ }^{4}$.

$\mathrm{O}$ controle da hemorragia deve anteceder a abordagem da VBP. A dissecção desta deve ser cuidadosa a fim de evitar a sua devascularização ${ }^{3,4}$. A sutura da lesão deve ser feita sem tensão ${ }^{2,4}$. Nos pacientes instáveis as opções são: drenagem simples da cavidade ou a ligadura da $\mathrm{VBP}^{2}$. Nos pacientes estáveis portadores de lesões simples e proximais (antes da desembocadura do cístico) a rafia da lesão e drenagem com sonda em $\mathrm{T}$ costuma ser eficaz ${ }^{4}$. Nas lesões complexas a realização de coledocojejunostomia 
término-lateral em Y de Roux apoiada ou não com uma sonda uretral intraluminal ou de um dreno em $\mathrm{T}$ é o tratamento de escolha ${ }^{3,4}$. Entretanto devido ao pequeno calibre da VBP o índice de estenose é grande. Nas lesões da VBP localizadas na sua porção intrapancreática ou intramural, a ligadura do colédoco associada a uma colecistojejunostomia é um tratamento simples e eficaz, com pequena possibilidade de estenose ${ }^{1,4}$. Para esta técnica ter sucesso devemos ter certeza que o ducto cístico é pérvio e que a sua desembocadura está localizada antes da ligadura da $\mathrm{VBP}^{5}$. Teoricamente, a existência de um fundo cego na VBP poderia ocasionar o aparecimento de colangite; entretanto, a sua dilatação obrigatória é um dado favorável para nova reconstrução do trânsito biliar.

\begin{abstract}
The authors present a case of distal common bile duct injury. Ligation of the bile duct and a bypass cholecystojejunostomy were chosen as treatment. Diagnosis of blunt traumatic injury to the extrahepatic biliary ducts may be difficult due to the benign nature of initial bile peritonitis. Surgical treatment for associated abdominal injuries usually makes the diagnosis possible. One of the challenges in the treatment of these injuries relates to the small diameter of the, usually, normal common bile duct. Primary repair and $T$ tube drainage is the best option for non-complex injuries. End-to-end anastomosis and, preferentially, biliary-enteric anastomosis are the best surgical options for more complex injuries. Severe injuries have high complication rates, especially when the distal portion of the common bile duct is affected. Early leaks and late strictures are likely to develop in these situations. Cholecistojejunostomy and ligation of the injuried common bile duct are good surgical options for complex injuries. They carry a low complication rate and consequently low morbidity.
\end{abstract}

Key words: Common bile duct,:Extrahepatic biliary, injuries.

\section{REFERÊNCIAS}

1 - Bade PG, Thomson SR, Hirshberg A, et al. - Surgical options in traumatic injury to the extrahepatic biliary tract. BrJ Surg, 1989,76(3): 256-258.

2 - Ivatury RR, Rohman M, Nallathambi M et al. - The morbidity of injuries of the extra-hepatic biliary sistem. JTrauma, 1985,25910): 967-973.

3 - Posner MC, Moore EE. - Extrahepatic biliary tract injuries: operative management plan. J Trauma, 1985,25(9): 833-837.

4- Feliciano, D. - Lesões biliares como resultado de traumatismo contusos e penetrantes. Clin Cir Am Norte, 1994,74:951-962.
5 - Turney WH, Lee JP, Raju S. - Complete transection of the common bile duct due to blunt ranuma. Ann Surg, 1974,179(4): 440-444.

Endereço para correspondência:

Sizenando Vieira Starling

Rua Grão Mogol,698 apto 304. Sion.

Belo Horizonte. Minas Gerais.

CEP: 30310-010

E-mail: sizastarling@bol.com.br 